\title{
Testing neutrino magnetic moment in ionization of atoms by neutrino impact
}

\author{
K. A. Kouzakov ${ }^{+}$, A. I.Studenikin* , M. B. Voloshin ${ }^{\dagger \S 1)}$ \\ + Department of Nuclear Physics and Quantum Theory of Collisions, Faculty of Physics, Moscow State University, Moscow 119991, \\ Russia \\ *Department of Theoretical Physics, Faculty of Physics, Moscow State University, Moscow 119991, Russia \\ ${ }^{\dagger}$ William I. Fine Theoretical Physics Institute, University of Minnesota, Minneapolis, Minnesota 55455, USA \\ $\S$ Institute of Theoretical and Experimental Physics, Moscow 117218, Russia
}

Submitted April 20, 2022

\begin{abstract}
The atomic ionization processes induced by scattering of neutrinos play key roles in the experimental searches for a neutrino magnetic moment. Current experiments with reactor (anti)neutrinos employ germanium detectors having energy threshold comparable to typical binding energies of atomic electrons, which fact must be taken into account in the interpretation of the data. Our theoretical analysis shows that the so-called stepping approximation to the neutrino-impact ionization is well applicable for the lowest bound Coulomb states, and it becomes exact in the semiclassical limit. Numerical evidence is presented using the Thomas-Fermi model for the germanium atom.
\end{abstract}

The neutrino magnetic moments (NMM) expected in the Standard Model are very small and proportional to the neutrino masses [1]: $\mu_{\nu} \approx 3 \times 10^{-19} \mu_{B}\left(m_{\nu} / 1 \mathrm{eV}\right)$ 'with $\mu_{B}=e / 2 m$ being the electron Bohr magneton, and $m$ is the electron mass. Thus any larger value of $\mu_{\nu}$ can arise only from physics beyond the Standard Model (a recent review of this subject can be found in Ref. 2]). Current direct experimental searches [3, 4, 5] for a magnetic moment of the electron (anti)neutrinos from reactors have lowered the upper limit on $\mu_{\nu}$ down to $\mu_{\nu}<3.2 \times 10^{-11} \mu_{B}[5]$. These ultra low background 'experiments use germanium crystal detectors exposed to the neutrino flux from a reactor and search for scat'tering events by measuring the energy $T$ deposited by the neutrino scattering in the detector. The sensitivity of such a search to NMM crucially depends on lower'ing the threshold for the energy transfer $T$, due to the 'enhancement of the magnetic scattering relative to the standard electroweak one at low $T$. Namely, the differential cross section $d \sigma / d T$ is given by the incoherent sum of the magnetic and the standard cross section, and for the scattering on free electrons the NMM contribution is given by the formula [6, 7]

$$
\frac{d \sigma_{(\mu)}}{d T}=4 \pi \alpha \mu_{\nu}^{2} \frac{1}{T}\left(1-\frac{T}{E_{\nu}}\right),
$$

where $E_{\nu}$ is the energy of the incident neutrino, and displays a $1 / T$ enhancement at low energy transfer. The

\footnotetext{
1) e-mail: voloshin@umn.edu
}

standard electroweak contribution is constant in $T$ at $E_{\nu} \gg T$ :

$$
\begin{array}{r}
\frac{d \sigma_{E W}}{d T}=\frac{G_{F}^{2} m}{2 \pi}\left(1+4 \sin ^{2} \theta_{W}+8 \sin ^{4} \theta_{W}\right) \times \\
\times\left[1+O\left(\frac{T}{E_{\nu}}\right)\right] \approx 10^{-47} \frac{\mathrm{cm}^{2}}{\mathrm{keV}}
\end{array}
$$

In what follows we refer to these two types of contribution to the scattering as, respectively, the magnetic and the weak.

The current experiments have reached threshold values of $T$ as low as few $\mathrm{keV}$ and are likely to further improve the sensitivity to low energy deposition in the detector. At low energies however one can expect a modification of the free-electron formulas (11) and (2) due to the binding of electrons in the germanium atoms, where e.g. the energy of the $K_{\alpha}$ line, $9.89 \mathrm{keV}$, indicates that at least some of the atomic binding energies are comparable to the already relevant to the experiment values of $T$. Thus, a proper treatment of the atomic effects in neutrino scattering is necessary and important for the analysis of the current and even more of the future data with a still lower threshold. For the first time this problem was addressed in Ref. [8], where a 2-3 times enhancement of the electroweak cross section in the case of ionization from a $1 s$ state of a hydrogenlike atom with nuclear charge $Z$ had been numerically determined at neutrino energies $E_{\nu} \sim \alpha Z m c^{2}$. Subsequent numerical calculations within the Hartree-FockDirac method for ionization from inner shells of various 
atoms showed much lower enhancement $(\sim 5-10 \%)$ of the electroweak contribution 9, 10, 11. The interest to the role of atomic effects was renewed in several recent papers, which however are ridden by a 'trial and error' approach. The early claim [12 of a significant enhancement of the NMM contribution by the atomic effects has been later disproved [13, 14] and it was argued [13, 15] that the modification of the formulas (1) and (2) by the atomic binding effects is insignificant down to very low values of $T$. It has been subsequently pointed out [15] that the analysis of Ref. 13] is generally invalidated in multi-electron systems, including atoms with $Z>1$. Furthermore, the analysis of Ref. [13] is also generally invalidated by singularities of the relevant correlation function in the complex plane of momentum transfer ${ }^{2)}$, so that the claimed behavior of the cross section at low $T$ applies only in the semiclassical limit.

In this paper we revisit the subject of neutrino scattering on atoms at low energy transfer. We aim at describing this process at $T$ in the range of few $\mathrm{keV}$ and lower, so that the motion of the electrons is considered as strictly nonrelativistic. Also in this range the energy of the dominant part of the incident neutrinos from the reactor is much larger than $T$ and we thus neglect any terms whose relative value is proportional to $T / E_{\nu}$. Furthermore any recoil of the germanium atom as a whole results in an energy transfer less than $2 E_{\nu}^{2} / M_{G e}$, which at the typical reactor neutrino energy is well below the considered here keV range of the energy transfer. Thus we formally set the mass of the atomic nucleus to infinity and neglect any recoil by the atom as a whole. In particular, under these conditions the interaction of the neutrino with the nucleus can be entirely neglected, and only the scattering on the atomic electrons is to be considered.

The kinematics of the scattering of a neutrino on atomic electrons is generally characterized by the components of the four-momentum transfer, the energy transfer $T$ and the spatial momentum transfer $\mathbf{q}$, from the neutrino to the electrons with two rotationally invariant variables being $T$ and $q=|\mathbf{q}|$. At small $T$ the electrons can be treated nonrelativistically both in the initial and the final state, so that the process is that of scattering of an NMM in the electromagnetic field $A=\left(A_{0}, \mathbf{A}\right)$ of the electrons: $A_{0}(\mathbf{q})=\sqrt{4 \pi \alpha} \rho(\mathbf{q}) / q^{2}$, $\mathbf{A}(\mathbf{q})=\sqrt{4 \pi \alpha} \mathbf{j}(\mathbf{q}) / q^{2}$, where $\rho(\mathbf{q})$ and $\mathbf{j}(\mathbf{q})$ are the Fourier transforms of the electron number density and current density operators, respectively,

\footnotetext{
2) The flaws in the momentum-transfer dispersion relation and sum rules of Ref. 13] are corrected in Ref. [18.
}

$$
\begin{aligned}
& \rho(\mathbf{q})=\sum_{a=1}^{Z} \exp \left(i \mathbf{q} \mathbf{r}_{a}\right) \\
& \mathbf{j}(\mathbf{q})=-\frac{i}{2 m} \sum_{a=1}^{Z}\left[\exp \left(i \mathbf{q} \mathbf{r}_{a}\right) \frac{\partial}{\partial \mathbf{r}_{a}}+\frac{\partial}{\partial \mathbf{r}_{a}} \exp \left(i \mathbf{q} \mathbf{r}_{a}\right)\right]
\end{aligned}
$$

and the sums run over the positions $\mathbf{r}_{a}$ of all the $Z$ electrons in the atom.

In this limit the expression for the double differential cross section is given by 15

$$
\begin{aligned}
\frac{d^{2} \sigma_{(\mu)}}{d T d q^{2}}=4 \pi \alpha \frac{\mu_{\nu}^{2}}{q^{2}} & {\left[\left(1-\frac{T^{2}}{q^{2}}\right) S\left(T, q^{2}\right)+\right.} \\
+ & \left.\left(1-\frac{q^{2}}{4 E_{\nu}^{2}}\right) R\left(T, q^{2}\right)\right],
\end{aligned}
$$

where $S\left(T, q^{2}\right)$, also known as the dynamical structure factor [16], and $R\left(T, q^{2}\right)$ are

$$
\begin{aligned}
& S\left(T, q^{2}\right)=\sum_{n} \delta\left(T-E_{n}+E_{0}\right)|\langle n|\rho(\mathbf{q})| 0\rangle|^{2}, \\
& R\left(T, q^{2}\right)=\sum_{n} \delta\left(T-E_{n}+E_{0}\right)\left|\left\langle n\left|j_{\perp}(\mathbf{q})\right| 0\right\rangle\right|^{2},
\end{aligned}
$$

with $j_{\perp}$ being the $\mathbf{j}$ component perpendicular to $\mathbf{q}$ and parallel to the scattering plane, which is formed by the incident and final neutrino momenta. The sums in Eqs. (6) and (7) run over all the states $|n\rangle$ with energies $E_{n}$ of the electron system, with $|0\rangle$ being the initial state.

Clearly, the factors $S\left(T, q^{2}\right)$ and $R\left(T, q^{2}\right)$ are related to respectively the density-density and current-current Green's functions

$$
\begin{aligned}
F\left(T, q^{2}\right) & =\sum_{n} \frac{|\langle n|\rho(\mathbf{q})| 0\rangle|^{2}}{T-E_{n}+E_{0}-i \epsilon} \\
& =\left\langle 0\left|\rho(-\mathbf{q}) \frac{1}{T-H+E_{0}-i \epsilon} \rho(\mathbf{q})\right| 0\right\rangle, \\
L\left(T, q^{2}\right) & =\sum_{n} \frac{\left|\left\langle n\left|j_{\perp}(\mathbf{q})\right| 0\right\rangle\right|^{2}}{T-E_{n}+E_{0}-i \epsilon} \\
& =\left\langle 0\left|j_{\perp}(-\mathbf{q}) \frac{1}{T-H+E_{0}-i \epsilon} j_{\perp}(\mathbf{q})\right| 0\right\rangle,
\end{aligned}
$$

as

$$
\begin{aligned}
& S\left(T, q^{2}\right)=\frac{1}{\pi} \operatorname{Im} F\left(T, q^{2}\right), \\
& R\left(T, q^{2}\right)=\frac{1}{\pi} \operatorname{Im} L\left(T, q^{2}\right),
\end{aligned}
$$

with $H$ being the Hamiltonian for the system of electrons. For small values of $q$, in particular, such that $q \sim T$, only the lowest-order non-zero terms of the expansion of Eqs. (10) and (11) in powers of $q^{2}$ are of 
relevance (the so-called dipole approximation). In this case, one has 15

$$
R\left(T, q^{2}\right)=\frac{T^{2}}{q^{2}} S\left(T, q^{2}\right) .
$$

Taking into account Eq. (12), the experimentally measured single-differential inclusive cross section is, to a good approximation, given by (see e.g. in Refs. [13. 15])

$$
\frac{d \sigma_{(\mu)}}{d T}=4 \pi \alpha \mu_{\nu}^{2} \int_{T^{2}}^{4 E_{\nu}^{2}} S\left(T, q^{2}\right) \frac{d q^{2}}{q^{2}} .
$$

The standard electroweak contribution to the cross section can be similarly expressed in terms of the same factor $S\left(T, q^{2}\right)[13$ as

$$
\begin{array}{r}
\frac{d \sigma_{E W}}{d T}=\frac{G_{F}^{2}}{4 \pi}\left(1+4 \sin ^{2} \theta_{W}+8 \sin ^{4} \theta_{W}\right) \times \\
\times \int_{T^{2}}^{4 E_{\nu}^{2}} S\left(T, q^{2}\right) d q^{2},
\end{array}
$$

where the factor $S\left(T, q^{2}\right)$ is integrated over $q^{2}$ with a unit weight, rather than $q^{-2}$ as in Eq. (13).

The kinematical limits for $q^{2}$ in an actual neutrino scattering are explicitly indicated in Eqs. (13) and (14). At large $E_{\nu}$, typical for the reactor neutrinos, the upper limit can in fact be extended to infinity, since in the discussed here nonrelativistic limit the range of momenta $\sim E_{\nu}$ is indistinguishable from infinity. The lower limit can be shifted to $q^{2}=0$, since the contribution of the region of $q^{2}<T^{2}$ can be expressed in terms of the photoelectric cross section 13 and is negligibly small (at the level of below one percent in the considered range of $T$ ). For this reason we henceforth discuss the momentumtransfer integrals in Eqs. (13) and (14) running from $q^{2}=0$ to $q^{2}=\infty$ :

$$
\begin{aligned}
& I_{1}(T)=\int_{0}^{\infty} S\left(T, q^{2}\right) \frac{d q^{2}}{q^{2}}, \\
& I_{2}(T)=\int_{0}^{\infty} S\left(T, q^{2}\right) d q^{2} .
\end{aligned}
$$

For a free electron, which is initially at rest, the density-density correlator is the free particle Green's function

$$
F_{(F E)}\left(T, q^{2}\right)=\left(T-\frac{q^{2}}{2 m}-i \epsilon\right)^{-1}
$$

so that the dynamical structure factor is given by $S_{(F E)}\left(T, q^{2}\right)=\delta\left(T-q^{2} / 2 m\right)$, and the discussed here integrals are in the free-electron limit as follows:

$$
\begin{aligned}
& I_{1}^{(F E)}=\int_{0}^{\infty} S_{(F E)}\left(T, q^{2}\right) \frac{d q^{2}}{q^{2}}=\frac{1}{T}, \\
& I_{2}^{(F E)}=\int_{0}^{\infty} S_{(F E)}\left(T, q^{2}\right) d q^{2}=2 m .
\end{aligned}
$$

It is readily seen that these expressions, when used in the formulas (13) and (14), result in the free-electron cross section in Eqs. (1) and (2).

Let us consider the scattering on just one bound electron. The Hamiltonian for the electron has the form $H=p^{2} / 2 m+V(r)$, and the density-density Green's function from Eq. (8) can be written as

$$
\begin{aligned}
F\left(T, q^{2}\right) & =\left\langle 0\left|e^{-i \mathbf{q r}}\left[T-H+E_{0}\right]^{-1} e^{i \mathbf{q r}}\right| 0\right\rangle \\
& =\left\langle 0\left|\left[T-\frac{q^{2}}{2 m}-\frac{\mathbf{p q}}{m}-H+E_{0}\right]^{-1}\right| 0\right\rangle
\end{aligned}
$$

where the infinitesimal shift $T \rightarrow T-i \epsilon$ is implied.

Clearly, a nontrivial behavior of the latter expression in Eq. (18) is generated by the presence of the operator $\mathbf{p q} / m$ in the denominator, and the fact that it does not commute with the Hamiltonian $H$. Thus an analytical calculation of the Green's function as well as the dynamical structure factor and the momentum-transfer integrals is feasible in only few specific problems. In particular, such a calculation for ionization from the $1 \mathrm{~s}$, $2 s$, and $2 p$ hydrogen-like states shows that the deviation of the discussed integrals (15) from their free values are very small 18: the largest deviation is exactly at the ionization threshold, where, for instance, each of the $1 \mathrm{~s}$ integrals is equal to the free-electron value multiplied by the factor $\left(1-7 e^{-4} / 3\right) \approx 0.957$, and in the $2 s$ and $2 p$ cases the departure from the free-electron behavior is even smaller.

The problem of calculating the integrals (15) however can be solved in the semiclassical limit, where one can neglect the noncommutativity of the momentum p with the Hamiltonian, and rather treat this operator as a number vector. Taking also into account that $\left(H-E_{0}\right)|0\rangle=0$, one can then readily average the latter expression in Eq. (18) over the directions of $\mathbf{q}$ and find the formula for the dynamical structure factor:

$$
\begin{aligned}
& S\left(T, q^{2}\right)=\frac{m}{2 p q} {\left[\theta\left(T-\frac{q^{2}}{2 m}+\frac{p q}{m}\right)-\right.} \\
&\left.-\theta\left(T-\frac{q^{2}}{2 m}-\frac{p q}{m}\right)\right],
\end{aligned}
$$

where $p=|\mathbf{p}|$ and $\theta$ is the standard Heaviside step function. The expression in Eq. (19) is nonzero only in the range of $q$ satisfying the condition $-p q / m<$ $T-q^{2} / 2 m<p q / m$, i.e. between the (positive) roots of the binomials in the arguments of the step functions: $q_{\min }=\sqrt{2 m T+p^{2}}-p$ and $q_{\max }=\sqrt{2 m T+p^{2}}+p$. One can notice that the previously mentioned 'spread 
and shift' of the peak in the dynamical structure function in this limit corresponds to a flat pedestal between $q_{\min }$ and $q_{\max }$. The calculation of the integrals (15) with the expression (19) is straightforward, and yields the free-electron expressions (17) for the discussed here integrals in the semiclassical (WKB) limit:

$$
I_{1}^{(W K B)}=\frac{1}{T}, \quad I_{2}^{(W K B)}=2 m .
$$

The difference from the pure free-electron case however is in the range of the energy transfer $T$. Namely, the expressions (20) are applicable in this case only above the ionization threshold, i.e. at $T \geq\left|E_{0}\right|$. Below the threshold the electron becomes 'inactive'.

It is instructive to point out that the validity of the result in Eq. (20) is based on the semiclassical approximation and is not directly related to the value of the energy $T$. In particular, for a Coulomb interaction the WKB approximation is applicable at energy near the threshold [17. For $T$ exactly at the threshold, $T=-E_{0}$, the criterion for applicability of the semiclassical approach in terms of the force $F=|\mathbf{F}|=|[\mathbf{p}, H]|$ acting on the electron and the momentum $p$ of the electron is that [17] the ratio of the characteristic values $m F / p^{3}$ is small. For the excitation of a state with the principal number $n$ one has $|F|=\alpha / r^{2} \sim m^{2} \alpha^{3} n^{4}$ and $p \sim m \alpha / n$, so that $m|F| / p^{3} \sim 1 / n$. Thus the applicability of a semiclassical treatment of the ionization near the threshold improves for initial states with large $n$. As previously mentioned, the modification of the integrals (15) by the binding is already less than $5 \%$ for $n=1$, so that we fully expect this deviation to be smaller for the higher states, and even smaller at larger values of $T$ above the threshold due to the approach to the freeelectron behavior at $T \gg E_{0}$.

We believe that the latter conclusion explains the so-called stepping behavior observed empirically [10] in the results of numerical calculations. Namely the calculated cross section $d \sigma / d T$ for ionization of an electron from an atomic orbital follows the free-electron dependence on $T$ all the way down to the threshold for the corresponding orbital with a very small, at most a few percent, deviation. This observation led the authors of Ref. 10] to suggest the stepping approximation for the ratio of the atomic cross section (per target electron) to the free-electron one:

$$
f(T) \equiv \frac{d \sigma / d T}{(d \sigma / d T)_{F E}}=\frac{1}{Z} \sum_{i} n_{i} \theta\left(T-\left|E_{i}\right|\right),
$$

where the sum runs over the atomic orbitals with the binding energies $E_{i}$ and the filling numbers $n_{i}$. Clearly, the factor $f(T)$ simply counts the fraction of 'active' electrons at the energy $T$, i.e. those for which the ionization is kinematically possible. For this reason we refer to $f(t)$ as an activation factor.

In considering the neutrino scattering on actual atoms one needs to evaluate the dependence of the number of active electrons on $T$. The energies of the inner $K, L$, and $M$ orbitals in the germanium atom are well known (see e.g. Ref. [11 and references therein) and provide the necessary data for a description of the neutrino scattering by the stepping formula (21) down to the values of the energy transfer $T$ in the range of the binding of the $M$ electrons, i.e. at $T \gtrsim\left|E_{M}\right| \sim 0.1 \mathrm{keV}$. The corresponding steps in the activation factor are shown in Fig. 1 .

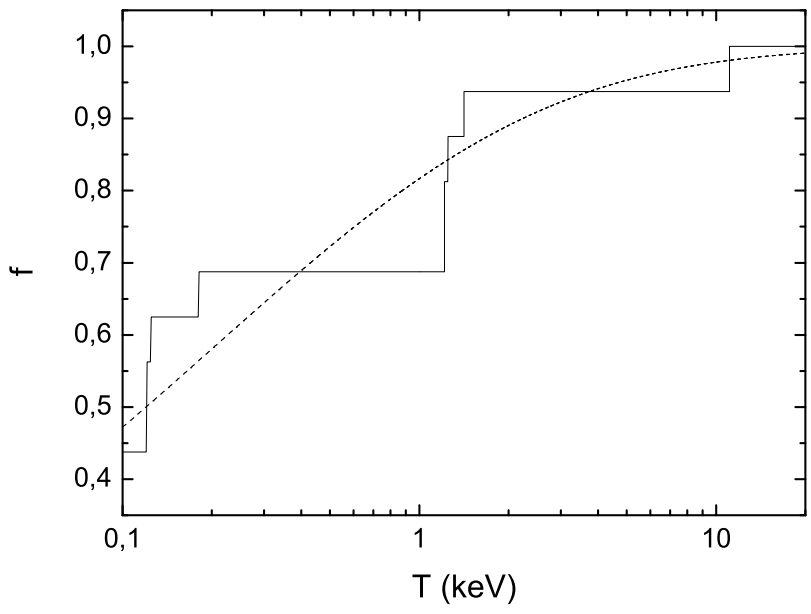

Fig. 1. The activation factor $f$ for germanium in the stepping approximation with the actual energies of the orbitals (solid line) and its value in the Thomas-Fermi model (dashed).

Let us show that the stepping approximation (21) can also be explained in terms of quantum corrections to the activation factor given by the Thomas-Fermi atomic model (see e.g. Ref. [17]). The latter describes electrons in an atom as a degenerate free electron gas in a master potential $\phi(r)$ filling the momentum space up to the zero Fermi energy, i.e. up to the momentum $p_{0}(r)$ such that $p_{0}^{2} / 2 m-e \phi=0$. The electron density $n(r)=p_{0}^{3} /\left(3 \pi^{2}\right)$ then determines the potential $\phi(r)$ from the usual Poisson's equation, thus resulting in a self-consistent procedure. In the discussed picture at an energy transfer $T$ the ionization is possible only for the electrons whose energies in the potential are above $-T$, i.e. with momenta above $p_{T}(r)$ with $p_{T}^{2} / 2 m-e \phi=-T$. The electrons with lower energy are inactive. Calculating the density of the inactive electrons as $p_{T}^{3} /\left(3 \pi^{2}\right)$ and subtracting their total number from $Z$, one readily arrives at the formula 
for the activation factor, i.e. the effective fraction of the active electrons $Z_{\text {eff }} / Z$ as a function of $T$ :

$$
f(T)=\frac{Z_{\mathrm{eff}}(T)}{Z}=1-\int_{0}^{x_{0}(T)}\left[\frac{\chi(x)}{x}-\frac{T}{T_{0}}\right]^{3 / 2} x^{2} d x,
$$

where $\chi(x)$ is the Thomas-Fermi function, well known and tabulated, of the scaling variable $x=2(4 / 3 \pi)^{2 / 3} m \alpha Z^{1 / 3}$, the energy scale $T_{0}$ is given by

$$
T_{0}=2\left(\frac{4}{3 \pi}\right)^{2 / 3} m \alpha^{2} Z^{4 / 3} \approx 30.8 Z^{4 / 3} \mathrm{eV}
$$

and, finally, $x_{0}(T)$ is the point where the integrand becomes zero, i.e. corresponding to the radius beyond which all the electrons are active at the given energy $T$. The energy scale $T_{0}$ in germanium $(\mathrm{Z}=32)$ evaluates to $T_{0} \approx 3.1 \mathrm{keV}$. The activation factor for germanium calculated from the formula (22) is shown by the dashed line in the plot of Fig. 2. One can see that the stepping activation factor (21) mimics upon average over the energy intervals between the electron shells in germanium the Thomas-Fermi result. Thus, it can be considered as refinement of the latter due to account for the quantization of the electron binding energies.

It should be remarked that the discussed statistical model is known to approximate the average bulk properties of the atomic electrons with a relative accuracy $O\left(Z^{-2 / 3}\right)$ and as long as the essential distances $r$ satisfy the condition $Z^{-1} \ll m \alpha r \ll 1$, which condition in terms of the scaling variable $x$ reads as $Z^{-2 / 3} \ll x \ll$ $Z^{1 / 3}$. In terms of the formula (22) for the number of active electrons, the lower bound on the applicability of the model is formally broken at $T \sim Z^{2 / 3} T_{0}$, i.e. at the energy scale of the inner atomic shells. However the effect of the deactivation of the inner electrons is small, of order $Z^{-1}$ in comparison with the total number $Z$ of the electrons. On the other hand, at low $T$, including the most interesting region of $T \sim T_{0}$, the integral in Eq. (22) is determined by the range of $x$ of order one, where the model treatment is reasonably justified.

We have considered the scattering of neutrinos on electrons bound in atoms. Our main finding is that the differential over the energy transfer cross section given by the free-electron formulas (1) and (2) and the stepping behavior of the activation factor given by Eq. (21) provides a very accurate description of the neutrinoimpact ionization of a complex atom, such as germanium, down to quite low energy transfer. The deviation from this approximation due to the onset of the ionization near the threshold is less than $5 \%$ (of the height of the step) for the $K$ electrons, if one applies the analytical behavior of this onset that we find for the ground state of a hydrogen-like ion. We also find that the free-electron expressions for the cross section are not affected by the atomic binding effects in the semiclassical limit. For this reason we expect that the deviation of the actual onset from a step function at the threshold for ionization of higher atomic orbitals is even smaller than for the ground state, since the motion in the higher states is closer to the semiclassical limit. Thus, our analytical results explain the numerically determined behaviors of the electroweak and magnetic contributions to the neutrino-impact ionization of various atomic targets [9, 10, 11].

We thank A. S. Starostin and Yu. V. Popov for useful and stimulating discussions. The work of K.A.K. (in part) and A.I.S. is supported by RFBR grant 11-0201509-a. K.A.K. also acknowledges partial support from RFBR grant 11-01-00523-a. The work of M.B.V. is supported in part by the DOE grant DE-FG02-94ER40823.

1. K. Fujikawa and R. Shrock, Phys. Rev. Lett. 45, 963 (1980).

2. C. Giunti and A. Studenikin, Phys. Atom. Nucl. 72, 2089 (2009).

3. H. T. Wong et al., Phys. Rev. D 75, 012001 (2007).

4. A. G. Beda et al., Phys. Atom. Nucl. 70, 1873 (2007).

5. A. G. Beda et al., Phys. Part. Nucl. Lett. 7, 406 (2010).

6. G. V. Domogatskii and D. K. Nadezhin, Sov. J. Nucl. Phys. 12, 678 (1971) [Yad. Fiz. 12, 1233 (1970)].

7. P. Vogel and J. Engel, Phys. Rev. D 39, 3378 (1989).

8. Yu. V. Gaponov, Yu. L. Dobrynin, and V.I. Tikhonov, Sov. J. Nucl. Phys. 22, 170 (1975) [Yad. Fiz. 22, 328 (1975)].

9. V. Yu. Dobretsov, A. B. Dobrotsvetov, and S. A. Fayans, Sov. J. Nucl. Phys. 55, 1180 (1992) [Yad. Fiz. 55, 2126 (1992)].

10. V. I. Kopeikin et al., Phys. At. Nucl. 60, 1859 (1997) [Yad. Fiz. 60, 2032 (1997)].

11. S. A. Fayans, L. A. Mikaelyan and V. V. Sinev, Phys. Atom. Nucl. 64, 1475 (2000) [Yad. Fiz. 64, 1551 (2000).

12. H. T. Wong, H. B. Li and S. T. Lin, Phys. Rev. Lett. 105, 061801 (2010).

13. M. B. Voloshin, Phys. Rev. Lett. 105, 201801 (2010).

14. H. T. Wong, H.B. Li, and S.T. Lin, preprint arXiv:1001.2074 v3.

15. K. A. Kouzakov and A. I. Studenikin, Phys. Lett. B 696, 252 (2011).

16. L. Van Hove, Phys. Rev. 95, 249 (1954).

17. L.D. Landau and E. M. Lifshits, Quantum Mechanics (Non-relativistic Theory), Third Edition, Pergamon, Oxford, 1977.

18. K. A. Kouzakov, A.I. Studenikin, and M. V. Voloshin, preprint arXiv:1101.4878 (2011). 